\title{
A hepatic stem cell vaccine is superior to an embryonic stem cell vaccine in the prophylaxis and treatment of murine hepatocarcinoma
}

\author{
QI ZHENG* , YICHAO ZHENG* , JING CHEN, JIA YOU, YUEYONG ZHU, YURUI LIU and JIA JI JIANG \\ Center for Liver Disease, The First Affiliated Hospital of Fujian Medical University, Fuzhou, Fujian 350004, P.R. China
}

Received June 7, 2016; Accepted October 31, 2016

DOI: $10.3892 /$ or.2017.5381

\begin{abstract}
Stem cells and cancer cells express a common subset of antigens called oncofetal antigens. Theoretically, vaccination with stem cells is effective at boosting the preexisting anticancer immune response. Herein we describe the efficacy of two stem cell-based vaccines in the prophylaxis and treatment of subcutaneous hepatic tumors transplanted into mice. C57BL/6j mice were vaccinated weekly with either hepatic stem cells (HSCs) or embryonic stem cells (ESCs) for three weeks, followed by a subcutaneous challenge with Hepa 1-6 cells at one week (group 1) or four weeks (group 2) after vaccination. No tumor formation was observed in HSC-vaccinated mice when challenged within one week after vaccination (group 1), but tumors formed in $10 \%$ of mice in the ESC-vaccinated group and in $60 \%$ of mice in the unvaccinated group. When the long-term memory response was examined (group 2), only $10 \%$ of HSC-vaccinated mice and $20 \%$ of ESC-vaccinated mice developed macroscopic hepatocarcinomas compared to $60 \%$ of the unvaccinated mice. Besides their function as prophylactic vaccines, administration of either HSC or ESC could be a potential treatment for cancer. In mice with subcutaneous hepatocarcinomas, complete clearance of tumor burden was observed in $80 \%$ of mice receiving HSC vaccination, but $40 \%$ of ESC-vaccinated mice presented with tumors that did not increase in size over time. These data support that HSC is a superior vaccine candidate for durable antitumor protection in this hepatocarcinoma model.
\end{abstract}

\section{Introduction}

Liver cancer (or hepatocarcinoma) currently accounts for $7 \%$ of all cancer cases diagnosed worldwide, resulting in

Correspondence to: Dr Jia-Ji Jiang, Center for Liver Disease, The First Affiliated Hospital of Fujian Medical University, Fuzhou, Fujian 350004, P.R. China

E-mail: doctorjjj@sina.cn

*Contributed equally

Key words: hepatic stem cell, hepatic oval cell, embryonic stem cell, cancer, vaccine, hepatocarcinoma
$>600,000$ deaths each year $(1,2)$. Treatments for early-stage hepatocellular carcinoma (HCC) include tumor resection, liver transplantation and local ablation. However, only 30-40\% of early-stage liver cancer patients are eligible to receive these therapies, and half of them may suffer from tumor recurrence within 3 years of treatment (1-3). New therapeutic strategies that improve the prognosis of liver cancer are warranted, and immunotherapy is one alternative approach that is showing therapeutic promise.

Anticancer vaccination has been applied as an active immunotherapeutic strategy to stimulate specific and durable antitumor immunity, and vaccines have been used in the treatment of liver cancer or even prevention of cancer recurrence $(4,5)$. The rationale for vaccination against cancer is based on the presence of circulating $\mathrm{T}$ cells that are reactive towards specific cancer-associated antigens expressed by tumor cells $(6,7)$. In this respect, immunization with univalent cancer antigen such as $\alpha$-fetoprotein (AFP), carcinoembryonic antigen and glypican-3 (GPC3) peptide has been proved to boost the antitumor immune response, leading to the decreased tumor burden and increased overall survival in patients with HCC $(3,4,8,9)$. However, the rapid genesis of mutant with decreased expression of targeted antigen restrains the efficacy of univalent cancer vaccine. Until now, it is difficult to identify all these targeted antigens and select the most critical one for developing cancer vaccine. Thus, whole cell vaccine that contain all these targeted antigens was thought to induce more robust antitumor immune responses than the traditional univalent cancer vaccine.

Recent studies have shown that embryonic stem cells (ESCs) can boost the immune system when used as a wholecell cancer vaccine, and this stimulation leads to a reduction in tumor burden (10-14). ESCs and certain cancer cells express a specific subset of antigens called oncofetal antigens, and ESCs are effective at presenting these antigens to the immune system and eliciting an anticancer response (10-18). Murine models of lung, colon and ovarian cancer have confirmed the antitumor efficacy of ESC vaccination, but it is not known if immunization with ESCs will have a measurable impact on liver cancer. Some evidence suggests that liver cancer may arise from the aberrant activation of hepatic stem cells (HSCs)/ hepatic progenitor cells (HPCs), as well as share cellular and molecular phenotypes with HSC/HPCs $(19,20)$. Theoretically, 
HSC/HPCs may serve as attractive vaccine candidates that trigger T-cell responses as effectively as ESCs and drive an antitumor response that is specific, for liver cancer.

Collectively, both ESC and HSC/HPCs share cell surface antigens with liver cancer cells (16-20), and both cell types are potential cancer vaccine candidates that may generate antitumor immunity against liver cancer. For this reason, we compared the effectiveness of ESC and HSC whole-cell cancer vaccines in the prophylaxis and treatment of subcutaneous hepatic tumors transplanted into adult mice. Our results show that immunization with the HSC vaccine prevented the development of liver cancer for up to four weeks after vaccination. In addition, established subcutaneous tumors significantly shrank after HSC vaccination. Interestingly, ESC vaccine is less effective than HSC vaccine in prophylaxis and treatment of liver. These data support that HSC is a superior vaccine candidate for durable antitumor protection in this hepatocarcinoma model.

\section{Materials and methods}

Mice. Wild-type C57BL/6j mice were obtained from the Wushi Laboratory (http://www.fzzmsoft.com/xieli/index.asp) and maintained at the Laboratory Animal Center in Fujian Medical University according to standard guidelines. To induce development of hepatic oval cells (HOCs) in the liver, 4-week-old C57BL/6j mice were fed a choline-deficient, ethionine-supplemented (CDE) diet for three weeks as previously described (21). This diet was comprised of choline-deficient chow (Medicience Ltd.) and $0.15 \%$ (w/v) DL-ethionine (SigmaAldrich) added to the drinking water. Wild-type C57BL/6j mice were immunized at 10-12 weeks of age. All animal protocols were approved by the Animal Care Committee of Fujian Medical University.

Cell lines. HOCs were selected as the source of HSCs in our vaccination strategy. HOCs were isolated from C57BL/6j mice that were fed a three-week CDE diet, and the cells were cultured under standard conditions $\left(37^{\circ} \mathrm{C}, 5 \% \mathrm{CO}_{2}\right)$ as previously described with some modifications (22-24). Briefly, mice were anaesthetized, the abdominal cavity was opened, and the liver was perfused via the hepatic portal vein with two buffers: $50 \mathrm{ml}$ of EGTA buffer [0.5 mM EGTA (Sigma-

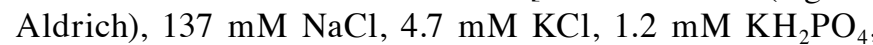
$0.65 \mathrm{mM} \mathrm{MgSO}_{4}, 10.07 \mathrm{mM}$ HEPES (Sigma-Aldrich), $\mathrm{pH} 7.4$ ], and $55 \mathrm{ml}$ of collagenase buffer [67 $\mathrm{mM} \mathrm{NaCl}, 6.7 \mathrm{mM} \mathrm{KCl}$, $4.76 \mathrm{mM} \mathrm{CaCl}_{2}, 100.7 \mathrm{mM}$ HEPES, $0.035 \%$ collagenase type I (Sigma-Aldrich), $\mathrm{pH} \mathrm{7.6]} \mathrm{at} \mathrm{a} \mathrm{flow} \mathrm{rate} \mathrm{of} 5 \mathrm{ml} / \mathrm{min}$. Next, the liver was removed, dissected, and digested in liver digestion buffer [0.1\% collagenase type VIII (Sigma-Aldrich), $0.09 \%$ Pronase (Roche Diagnostics), $0.025 \%$ trypsin $/ 0.01 \%$ EDTA, 0.004\% DNase (Sigma-Aldrich)] at $37^{\circ} \mathrm{C}$ for $50 \mathrm{~min}$. After incubation, an equal volume of cold Williams' $\mathrm{E}$ medium containing 2\% FBS was added to the digestion buffer, and the mixtures were filtered through a $40-\mu \mathrm{m}$ cell strainer (BD Biosciences). Cells were pelleted, re-suspended in phosphate-buffered saline (PBS), and separated by density gradient centrifugation at $1400 \mathrm{x}$ g for $20 \mathrm{~min}$. Cells were collected, washed and plated at a density of $1 \times 10^{6}$ viable cells $/ \mathrm{ml}$ onto collagen-coated dishes in Williams' E medium with $10 \%$ FBS (Gibco), $10 \mathrm{mM}$ nicotinamide (Sigma-Aldrich), $2 \mathrm{mM}$ glutamine (Solarbio), $10^{-7} \mathrm{M}$ dexamethasone, $1 \mathrm{X} \mathrm{ITS}{ }^{+}$ (Sigma-Aldrich), $0.2 \mathrm{mM}$ ascorbic acid (Sigma-Aldrich), $20 \mathrm{mM}$ HEPES, $1 \mathrm{mM}$ Na pyruvate (Sigma-Aldrich), $0.15 \%$ $\mathrm{NaHCO}_{3}, 14 \mathrm{mM}$ glucose, $20 \mathrm{ng} / \mathrm{ml}$ EGF (BD Biosciences), $1.0 \%(\mathrm{v} / \mathrm{v})$ fungizone (Solarbio), and $1.0 \%(\mathrm{v} / \mathrm{v})$ penicillin/ streptomycin (Solarbio). Cells were sub-cultured for 10 weeks (30-35 passages), until the HOCs became stable and displayed consistent morphology.

The C57BL/6 mouse embryonic stem cells (mESCs) were purchased from a commercial supplier (Cyagen Bioscience Inc.), and co-cultured with C57BL/6 mouse embryonic fibroblasts (MEFs) as feeder cells in Dulbecco's modified Eagle's medium (DMEM) supplemented with $15 \%$ fetal bovine serum (FBS), 2 mM L-glutamine, $0.1 \mathrm{mM}$ non-essential amino acids, $0.1 \mathrm{mM}$ 2-mercaptoethanol, and $1000 \mathrm{U} / \mathrm{ml}$ leukemia inhibitory factor. Preparation of MEF feeders was performed as previously described, with some modifications (25). Briefly, the MEFs were derived from C57BL/6 mouse embryos (around day 13 of gestation) and cultured in DMEM supplemented with $10 \%$ FBS. After cell propagation (2-3 passages), MEFs were mitotically inactivated by treatment with $10 \mu \mathrm{g} / \mathrm{ml}$ mitomycin $\mathrm{C}$ in MEF culture medium for $3 \mathrm{~h}$, plated onto untreated culture vessels at density of $3.0-4.0$ cells $/ \mathrm{cm}^{2}$, and used as feeder cells.

The hepatoma cell line of C57BL/6 origin (Hepa 1-6) was purchased from the Cell Bank of the Chinese Academy of Sciences (http://www.cellbank.org.cn/mulu.asp) and cultured under standard conditions in DMEM supplemented with $10 \%$ FBS as described above. At the time of inoculation, Hepa 1-6 and mESCs were in passages 5-10 and 10-15, respectively.

Preparation of HOC and mESC whole-cell vaccine. HOCs were removed from the dish by treatment with $0.25 \%$ trypsinEDTA whereas mESCs were detached and separated from MEFs using the 'differential adhesion method' as previously described (26). The HOC and mESC whole-cell vaccines were generated by fixing HOCs and mESCs in a solution of $4 \%$ paraformaldehyde for $1 \mathrm{~h}$, followed by three washes with sterile PBS. Finally the cells were re-suspended in PBS at a concentration of $1 \times 10^{6}$ cells $/ \mathrm{ml}$, stored at $4^{\circ} \mathrm{C}$, and used for vaccination within one week.

Immunization protocol and tumor challenge. Paraformaldehyde-fixed, whole-cell vaccines $\left(1 \times 10^{6}\right.$ HOCs or mESCs) and live hepatoma cells ( $4 \times 10^{6}$ Hepa 1-6 cells/mouse) were administered by subcutaneous inoculation in the mid-left and mid-right abdominal region of mice, respectively. The scheme of immunization and tumor inoculation was described elsewhere, with some modifications (Fig. 1) $(11,12)$. To determine and compare the efficacy of HSC or mESC as a whole-cell vaccine, naive C57BL/6j mice were vaccinated with either HOCs or mESCs three times in one-week intervals, followed by subcutaneous inoculation with Hepa 1-6 cells at one week after the last $\mathrm{HOC} / \mathrm{mESC}$ boost (Fig. 1A). For long-term memory responses, mice were challenged with Hepa 1-6 cells at four weeks after the last vaccination (Fig. 1B).

To determine and compare the therapeutic efficacy of HSC or mESC as a whole-cell vaccine (Fig. 1C), C57BL/6 mice 

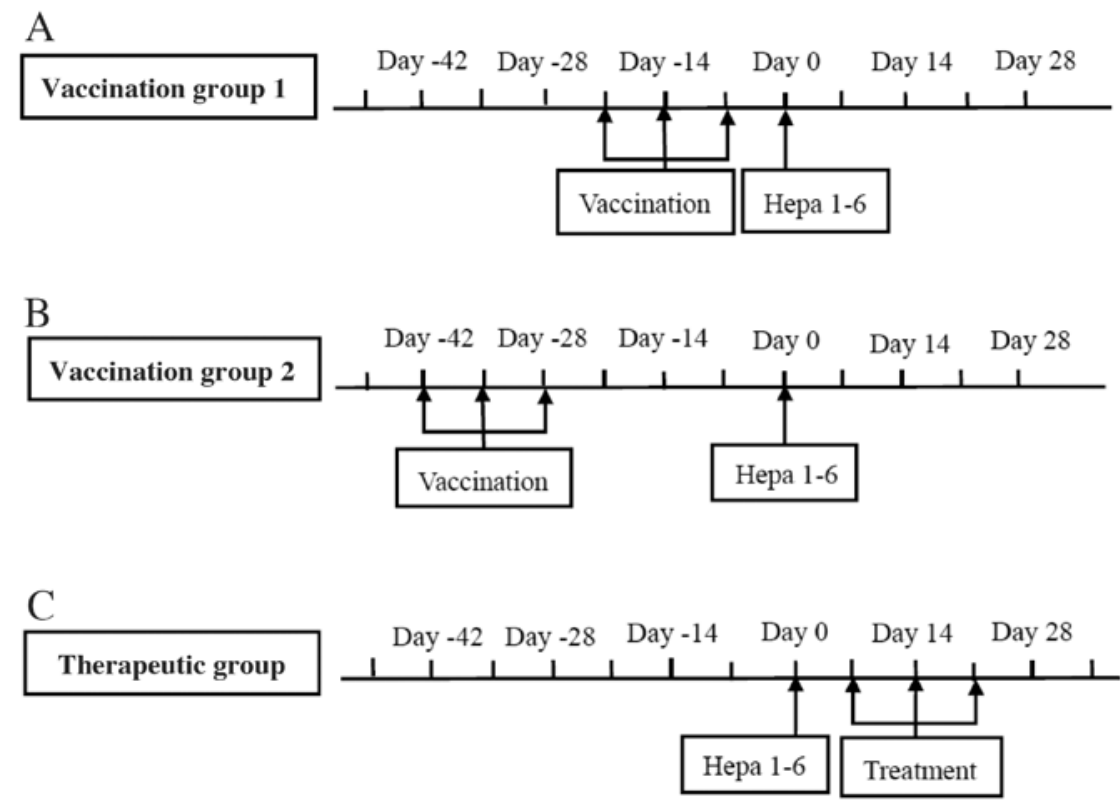

Figure 1. Scheme of immunization and tumor inoculation. (A) Mice were immunized weekly for three weeks followed by tumor inoculation at one week after the last vaccination. (B) Mice were vaccinated weekly for three weeks followed by tumor inoculation at four weeks after the last vaccination. (C) Mice received immunization three times (at one-week interval) 7 days after tumor inoculation.

Table I. The modified WHO criteria.

Response criteria

$\mathrm{CR}$

PR

SD

$\mathrm{PD}$

\section{Complete disappearance of all lesions}

At least 50\% decrease in tumor burden compared with baseline

Neither sufficient shrinkage to qualify for PR nor sufficient increase to qualify for PD

At least $25 \%$ increase in tumor burden compared with baseline received immunization three times (at one-week interval) 7 days after tumor inoculation. As a control, mice were inoculated with Hepa 1-6 without vaccination or treatment. Tumor growth was monitored every other day using digital calipers to measure both the longitudinal ( $\mathrm{L}, \mathrm{mm}$ ) and transverse diameters ( $\mathrm{T}, \mathrm{mm}$ ). Tumor area $\left(\mathrm{LxT}, \mathrm{mm}^{2}\right.$ ) was calculated as previously described (10). Mice were also monitored for general health indicators after immunization, such as overall behavior, feeding, body weight and ruffled fur. If the tumor diameter exceeded $15 \mathrm{~mm}$, or if tumor ulceration was observed, the mice were euthanized. Per our approved protocol that was primarily designed to evaluate the tumor formation rate of mice and response rate and to ease the pain and suffering of tumor-bearing mice, no survival experiments were conducted in this study.

Indirect immunofluorescence cytochemistry. Isolated HOCs were confirmed by immunofluorescence analysis and testing for the expression of two commonly associated HOC markers: cytokeratin 19 (CK19) and muscle pyruvate kinase $2\left(\mathrm{M}_{2} \mathrm{PK}\right)$. Briefly, cells were harvested from culture dishes and grown on poly-L-lysine-coated coverslips. Cells were fixed with $4 \%$ paraformaldehyde at room temperature for 20-30 min and then permeabilized with $0.5 \%$ Triton X-100 for 5 min. After permeabilization, cells were blocked with $1 \%$ bovine serum albumin (BSA) and incubated with the following primary anti- bodies at $4^{\circ} \mathrm{C}$ overnight: goat anti-CK19 $(1 \mu \mathrm{g} / \mathrm{ml}$, Santa Cruz Biotechnology), or rabbit anti-M ${ }_{2} \mathrm{PK}(1 \mu \mathrm{g} / \mathrm{ml}$, Abcam). Next, cells were washed three times in PBS and incubated with a secondary donkey anti-goat CruzFluor ${ }^{\mathrm{TM}} 594$ antibody (1 $\mu \mathrm{g} / \mathrm{ml}$, Santa Cruz Biotechnology), or donkey anti-rabbit Alexa Fluor ${ }^{\circledR} 488$ antibody $(2 \mu \mathrm{g} / \mathrm{ml}$, Abcam), for $30 \mathrm{~min}$ at $37^{\circ} \mathrm{C}$. Finally cells were washed three more times, mounted in antifade mounting medium (Beyotime) with 4',6- diamidino2-phenylindole (DAPI), and examined under a fluorescence microscope (Carl-Zeiss Axiovert 200).

Statistical analysis. The statistical analysis was performed using SPSS statistical software 13.0 and GraphPad Prism 5.0 software. Antitumor responses to immunotherapeutic vaccination were evaluated using a modification of the WHO criteria (Table I). Comparison of the cancer formation rate between the prophylaxis groups, and the disease control rate [DCR: complete response $(\mathrm{CR})+$ partial response $(\mathrm{PR})+$ stable disease (SD)] between treatment groups, was made by the $\chi^{2}$ method. The P-value was adjusted to 0.0125 . The dynamic tumor growth size was analyzed using a multivariate linear model. After confirming the inequality of variance with the Levene test, the post-therapeutic change in tumor size was analyzed with the Wilcoxon test. Differences in tumor growth size and weight between groups were detected by the 

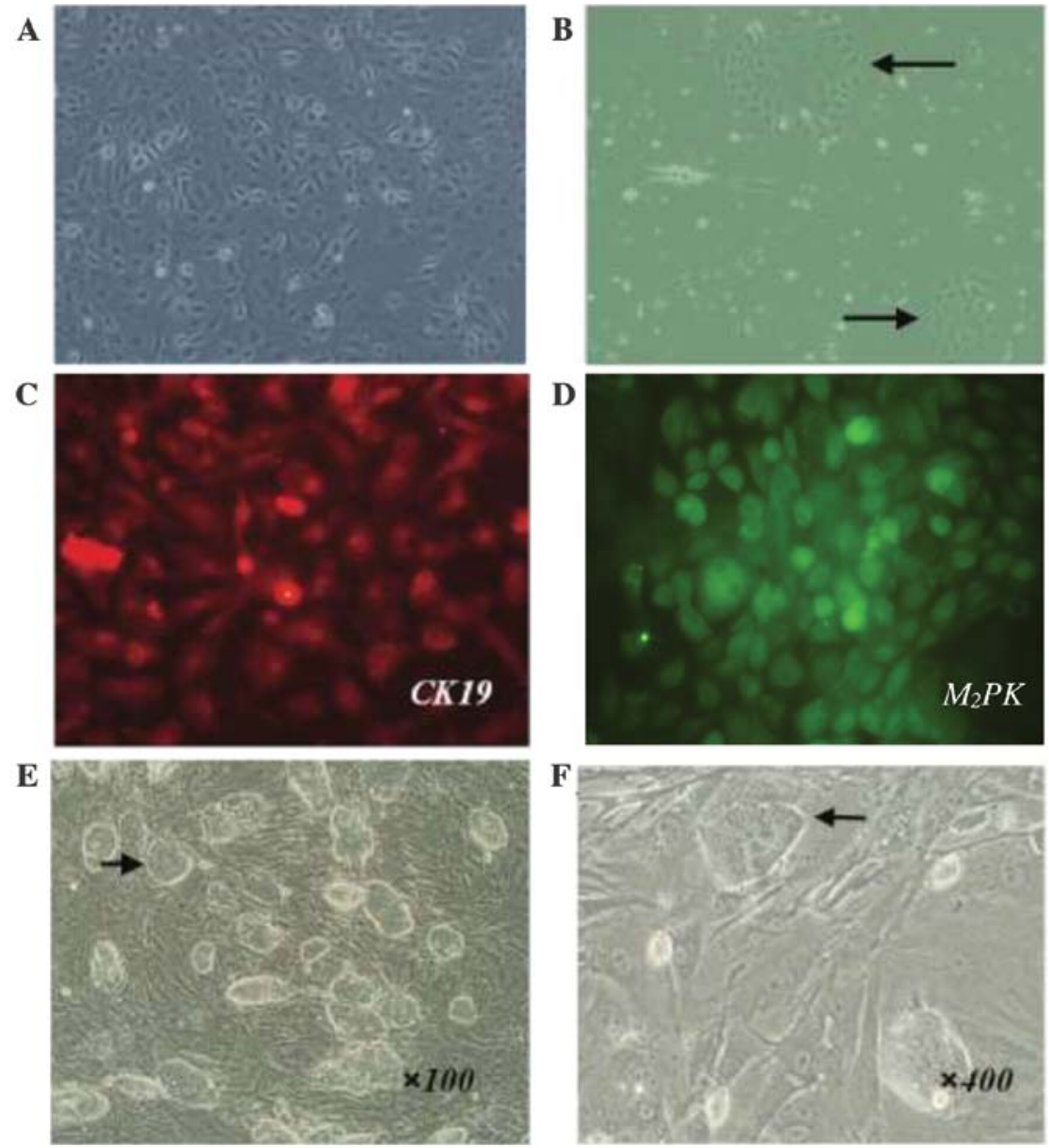

Figure 2. (A and B) The morphology of murine hepatic oval cells (HOCs) visualized by phase contrast microscopy (x100). The arrow indicates identifiable clones. (C and D) Immunofluorescence analysis of CK19 (C) and $\mathrm{M}_{2} \mathrm{PK}$ (D) expression (x400) on HOCs. (E and F) The morphology of embryonic stem cells (ESCs) visualized by phase-contrast microscopy at two magnifications [(E) x100; (F) x400]. The arrow indicates undifferentiated mESC clones.

Nemenyi-test. Most data were presented as mean \pm SEM, and a P-value $<0.05$ was considered statistically significant.

\section{Results}

Characterization of cultured HOCs and ESCs for vaccine preparation. In this study, HOCs were readily isolated from C57BL/6j mice by a two-step collagenase digestion and density gradient centrifugation. Following serial passage in vitro, HOC cell lines displayed consistent morphology (Fig. 2). HOCs were adherent, cobblestone-like cells with ovoid nuclei and scant cytoplasm. The majority of cells formed uniform monolayers (Fig. 2A), and a small number of cells produced identifiable clones (Fig. 2B). Vaccine preparation was performed after HOCs had been continuously cultured for $>3$ months. Following serial passaging (30-35 times), HOCs maintained viability, proliferative capacity, and typical cellular morphology. These results suggest that the HOCs were immortalized.

HOC cell lines derived from primary HOCs were further characterized by immunofluorescence staining for two commonly known murine oval cell markers: the hepatic progenitor and cholangiocyte marker (CK19), as well as the hepatic progenitor and early hepatocyte marker $\mathrm{M}_{2} \mathrm{PK}$. $\mathrm{HOC}$ cell lines were positive for expression of both CK19 and $\mathrm{M}_{2} \mathrm{PK}$, further suggesting that these HOCs were hepatic progenitor cells and ideal candidates for HSCs (Fig. 2C and D). Together these data show the establishment of a stable HOC cell line that can be used as a source of HSCs in a cancer vaccine.

To establish an ESC vaccine, we purchased mESCs (C57BL/6 origin) from a commercial supplier. The cells were maintained in an undifferentiated state by co-culturing them with MEFs (see Materials and methods). The undifferentiated mESC clones typically had clear borders, were round in appearance, and demonstrated a high nuclear-cytoplasmic ratio and prominent nucleoli (Fig. 2E and F).

HSC vaccination is superior to ESC vaccination in generating long-lasting antitumor protection against subcutaneously injected hepatic tumor cells. We compared the effectiveness of HSC and ESC whole-cell vaccination in the prophylaxis of subcutaneous hepatic tumor cells transplanted into adult mice (Fig. 3). Animals were challenged with live Hepa 1-6 cells shortly after receiving a series of immunizations, and tumor formation was monitored. At 31 days post-tumor challenge, the rate of tumor formation in the HSC vaccination group was $0 \%$ ( 0 out of 10 mice), $10 \%$ in the ESC vaccination group ( 1 out of 10 mice), and $60 \%$ in the control group (6 out of 10 mice; Fig. 3A). Tumor incidence was significantly increased in the control group compared to either the HSC or ESC vaccination 

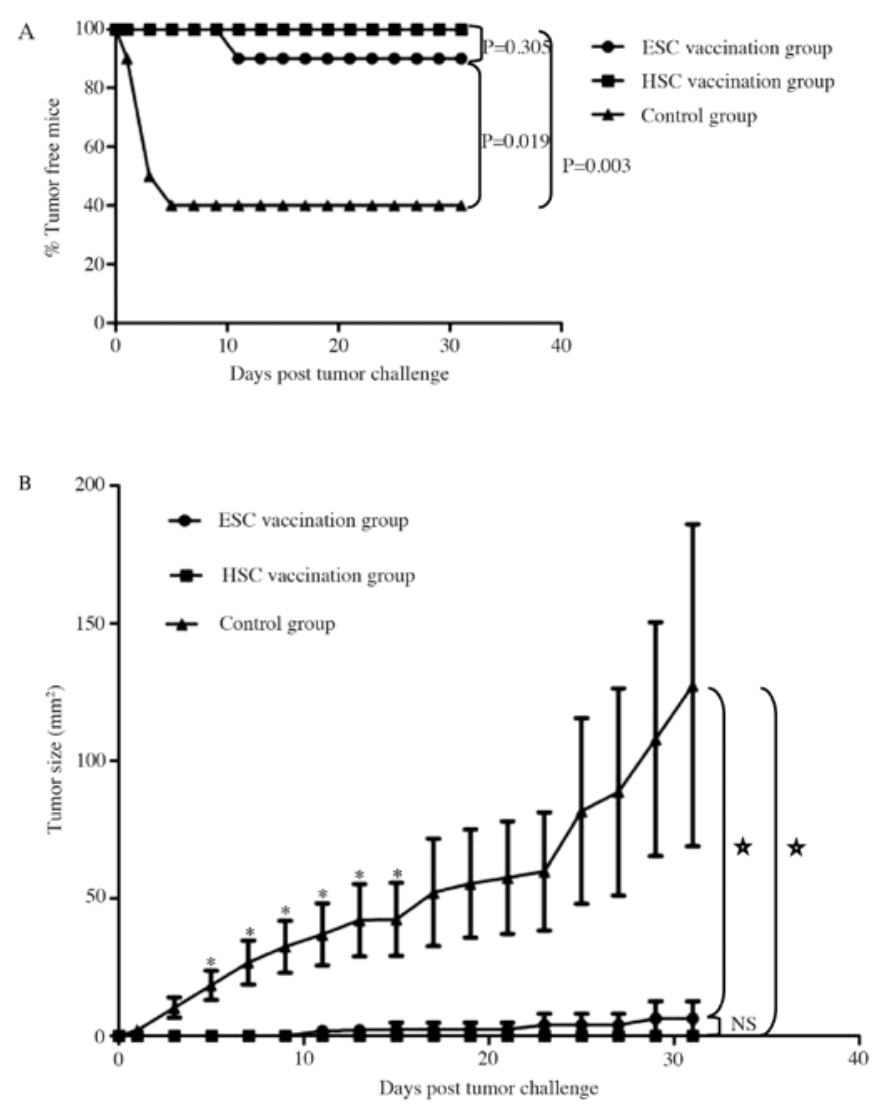

Figure 3. Comparison of HSC and ESC vaccines in the prophylaxis of subcutaneous hepatomas (short-term prevention). (A) Tumor formation in three groups of mice ( $\mathrm{N}=10$ per group) following vaccination. (B) Kinetics of tumor growth beginning at day 5 post-tumor challenge. An asterisk indicates a significant reduction in the average tumor size of the vaccination groups (HSC or ESC) compared to the control group $(\mathrm{P}<0.05)$. Bars represent mean \pm SEM.

group $(\mathrm{P}<0.05)$, but the rate of tumor incidence was similar between the HSC and ESC vaccination groups $(\mathrm{P}>0.05)$. Preventative vaccination significantly reduced tumor growth after day 5 (Fig. 3B; P<0.05). Hence both HSC and ESC vaccination effectively prevented the establishment of implantable hepatocarcinomas especially when the tumor challenge was administered within one week of the last immunization.

We further investigated if preventative immunization with HSC or ESC vaccines conferred long-lasting protection against a subsequent challenge with hepatocarcinoma cells (Fig. 4). A second challenge was administered by inoculating immunized mice with live Hepa 1-6 cells at four weeks after the last immunization. As shown in Fig. 4A, 90\% of mice immunized with the HSC whole-cell vaccine and $80 \%$ of mice that received the ESC whole-cell vaccine did not develop subcutaneous hepatocarcinomas for up to 31 days after the tumor challenge. Both the rate of tumor formation and the average tumor size were significantly reduced in the HSC vaccination group (Fig. 4B; $\mathrm{P}<0.05)$, but the ESC immunization group demonstrated a less robust decrease in tumor and tumor size $(\mathrm{P}>0.05)$. These results suggest that HSC vaccination was superior to ESC vaccination in generating long-lasting antitumor protection.

We confirmed these results by comparing the weight of subcutaneous hepatomas harvested from different groups at
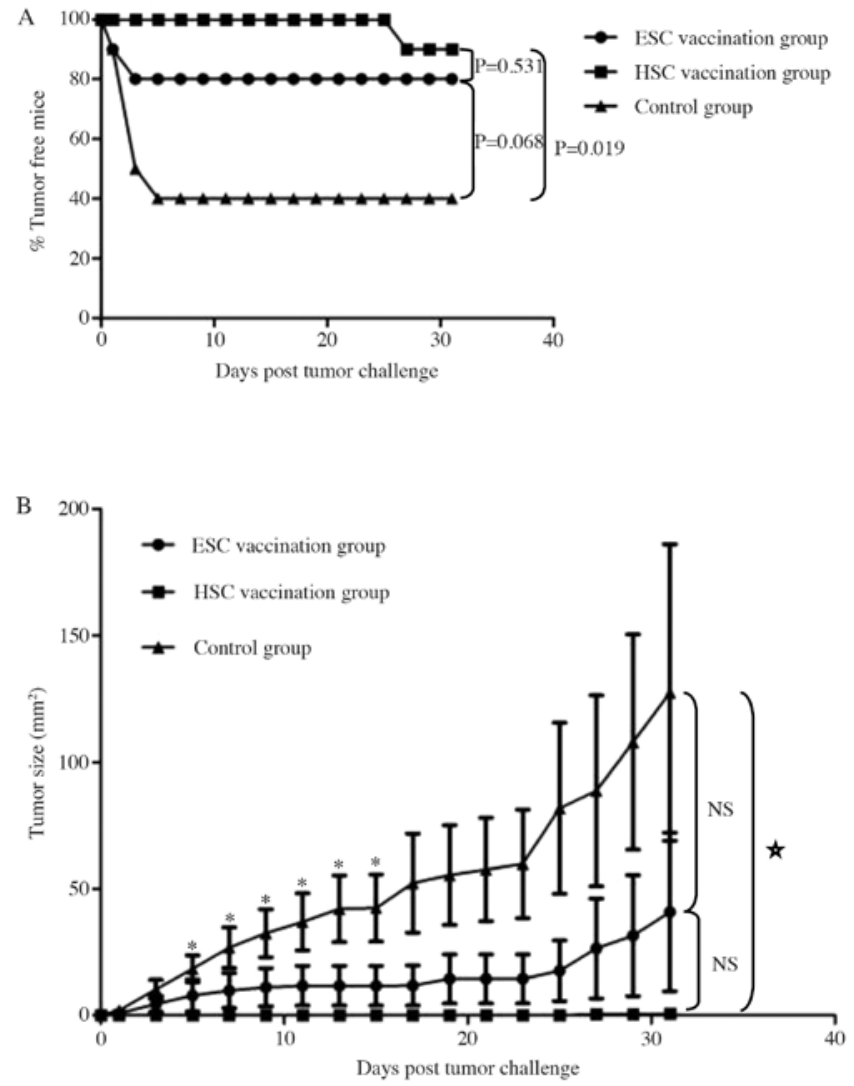

Figure 4. Comparison of HSC vaccine and ESC vaccines in the prophylaxis of subcutaneous hepatomas (long-lasting prevention). (A) Tumor formation and $(\mathrm{B})$ tumor size in three groups of mice $(\mathrm{N}=10)$ following vaccination. An asterisk indicates a significant reduction in the average tumor size of the HSC vaccination group compared to the control group $(\mathrm{P}<0.05)$. Bars represent mean \pm SEM.

31 days post-tumor challenge when most of subcutaneous tumor lesion became ulcerated. The average weight of the subcutaneous hepatomas was significantly reduced in the HSC vaccination group compared to the control group $(\mathrm{P}<0.05)$, but modestly decreased in both ESC vaccination groups compared with the control group ( $\mathrm{P}>0.05$; Fig. 5A). Macroscopic examination of subcutaneous hepatomas indicated that no mass was found in HSC vaccination group 1 while one mass was found in a mouse in ESC vaccination group 1, although the number of mice in each group was equal (10 mice per group) in this study. Furthermore, two of ten mice in ESC vaccination group 2 and one of ten mice in HSC vaccination group 2 developed subcutaneous hepatoma at the end of the study. Obviously, the size and weight of hepatoma developed in a mouse HSC vaccination group was significantly reduced compared with either ESC vaccination group or control group (Fig. 5). Collectively, our results indicate that HSC vaccination is better than ESC vaccination in the prophylaxis of implantable hepatocarcinomas in mice.

HSC vaccination is superior to ESC vaccination in the treatment of established hepatocarcinoma tumors in mice. Next, we investigated whether HSC or ESC whole-cell vaccination was effective at reducing tumor load in established hepatocarcinomas. Mice were inoculated with Hepa 1-6 cells, and solid tumors were established (day 5 post-inoculation). Mice were 


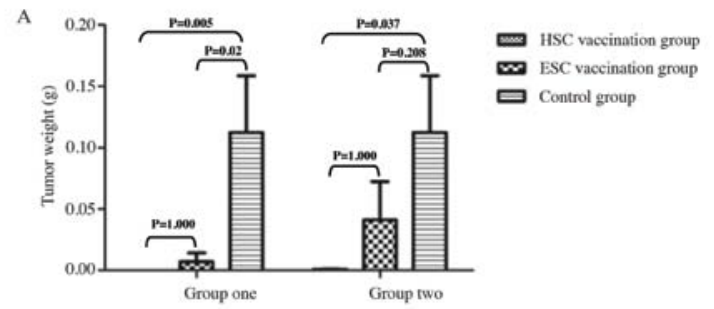

B

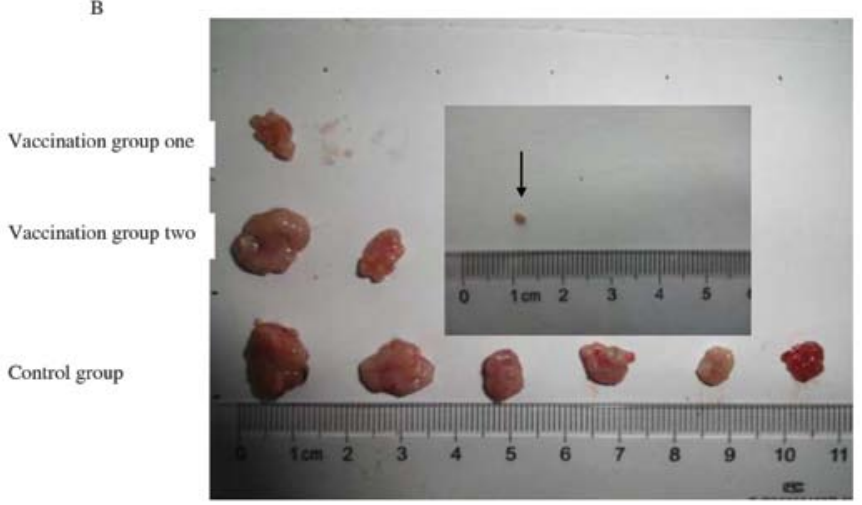

Figure 5. Macroscopic assessment of tumor burden in immunized mice. C57BL/6 mice were vaccinated weekly with either HSCs or ESCs for three weeks, followed by subcutaneous challenge with Hepa 1-6 cells at one week (group 1), or at four weeks (group 2) after vaccination. In the control group, mice were inoculated with Hepa 1-6 cells but did not receive any vaccination or treatment. (A) Comparison of the average weight of subcutaneous hepatomas harvested from each group at 31 days post-tumor challenge. A significant reduction in weight compared to the control group is indicated by $\mathrm{P}<0.05$. (B) The macroscopic assessment of subcutaneous hepatomas harvested from mice at day 31 post-challenge. The arrow indicates one subcutaneous hepatoma harvested from a mouse receiving the HSC vaccine (group 2).
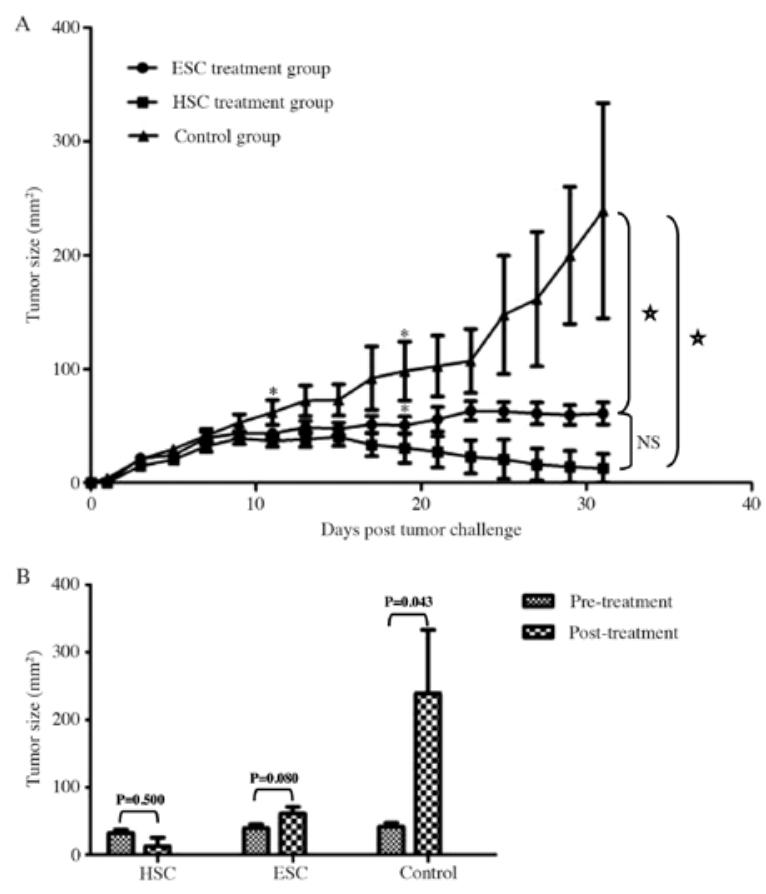

Figure 6. Comparison of HSC vaccination to ESC vaccination in the treatment of established hepatocarcinomas. (A) Kinetics of tumor growth. An asterisk indicates a significant reduction in the average size of the subcutaneous hepatocarcinomas compared to the control group $(\mathrm{P}<0.05)$. (B) Change in the size of subcutaneous hepatocarcinomas following treatment. The data represent the average tumor volumes of 5 mice from each group. Error bars represent mean \pm SEM.

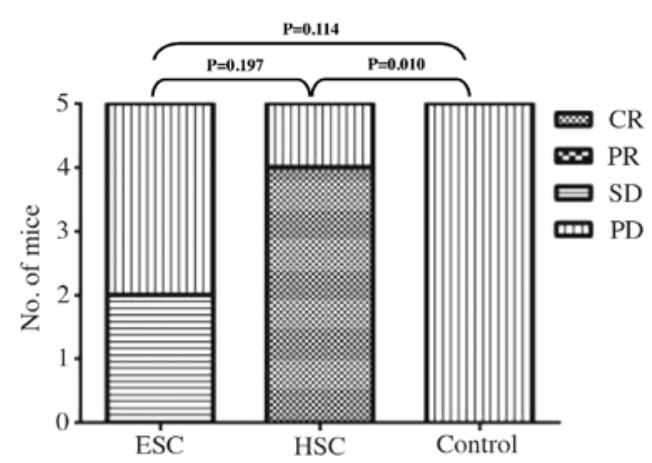

Figure 7. Evaluation of each treatment group according to the modified WHO criteria. The relative number of mice per group that had CR, PR, SD, PD following each treatment (control, HSC or ESC vaccination) are shown. Abbreviations are defined in Table I.
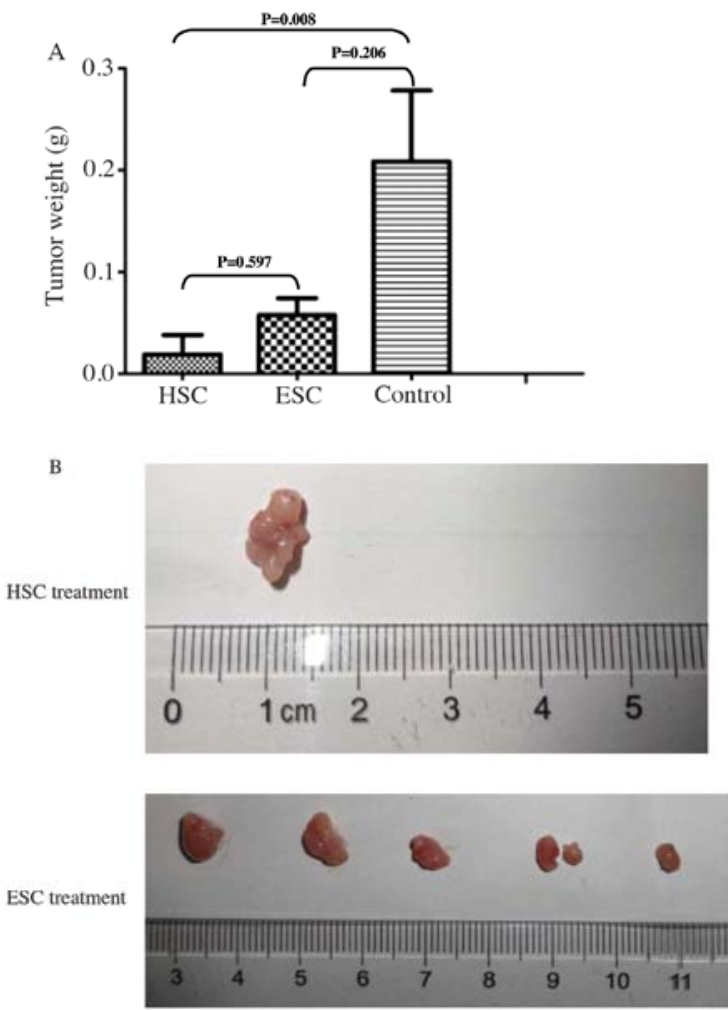

Control

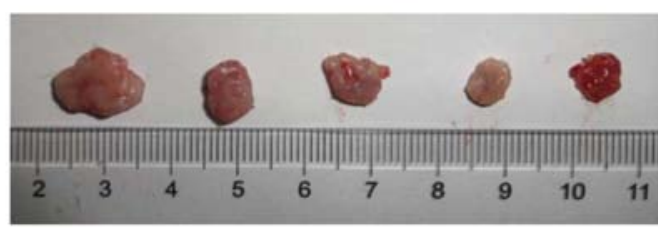

Figure 8. Weight and morphology of established hepatocarcinomas following HSC or ESC vaccination. (A) Comparison of the average weight of tumors harvested from each treatment group is shown. Bars represent mean \pm SEM. (B) Macroscopic assessment of established subcutaneous hepatocarcinomas harvested from mice at the termination of the experiment.

randomly divided into a control group (no vaccine), or into HSC and ESC vaccine treatment groups. No significant differences in the initial tumor burden or size were found between the three groups at the start of the treatment, as confirmed by the Wilcoxon signed-rank test $(\mathrm{P}>0.05)$. When tumors were examined at day six after the first vaccination, the average 
size of the subcutaneous hepatomas was significantly reduced in the HSC treatment group ( $\mathrm{P}<0.05$; Fig. $6 \mathrm{~A})$. Immunization with the ESC vaccine significantly reduced tumor growth at 14 days post-vaccination $(\mathrm{P}<0.05)$.

The subcutaneous hepatocarcinomas grew progressively in the control group, and most of the tumor lesions became ulcerated by the end of study (day 31). However, none of the mice developed obvious tumor ulceration in the vaccine treatment groups during this study (data not shown). Strikingly, the average size of the subcutaneous hepatocarcinomas continued to decline modestly following HSC treatment $(\mathrm{P}>0.05)$, although a slight increase in tumor volume was observed in mice receiving ESC treatment ( $\mathrm{P}>0.05$; Fig. 6).

We also evaluated the antitumor responses to immunotherapeutic vaccination using a modification of the WHO criteria (Table I). Although 1 out of 5 mice still suffered from progressive disease during HSC treatment, 4 out of 5 mice showed complete clearance of tumor burden following the three-week treatment (Fig. 7). In the ESC treatment group, 2 out of 5 mice presented with stable tumor loads, and 3 out of 5 mice succumbed to progressive disease by the end of study. In contrast, all of the mice developed progressive disease in the control group (Fig. 7).

All mice were euthanized at the end of this study, and the subcutaneous hepatocarcinomas were harvested and weighed. The average tumor weight decreased modestly in the HSC treatment group and was slightly reduced in the ESC treatment group, but any decline in tumor weight was not statistically significant ( $\mathrm{P}>0.05$; Fig. 8A). Since most of subcutaneous tumor lesions disappeared following the HSC vaccination, only one mass that was visible in one of the five mice in HSC treatment group could be harvested for further comparison. However, the tumor masses were all detectable in the five mice in ESC treatment group following the ESC vaccination. Macroscopic examination of the existing tumors revealed that the size of subcutaneous hepatomas reduced significantly in HSC vaccination group and decreased modestly in ESC vaccination group compared to the control group (Fig. 8B). Collectively, our results suggest that therapeutic immunization with HSCs is superior to ESCs in the treatment of established hepatocarcinomas in mice.

\section{Discussion}

The goal of activating the immune system for the prophylaxis and treatment of liver cancer has led to the investigation of several immunotherapeutic approaches, including cancer vaccines $(3,4,7)$. Since the antigens expressed by cancer cells are usually heterogeneous and plastic, traditional cancer vaccines that target single or multiple cancer-associated antigens may not be sufficient enough to eradicate all cancer cells (27). As knowledge about the nature of these targeted antigens expands, and as new antigenic oncoproteins are identified, the promise of developing an effective vaccine for the prevention and treatment of liver cancer is growing. In this respect, development of a stem cell-based vaccine is a good therapeutic strategy due to the shared expression of specific oncofetal antigens with liver cancer cells, as well as the ability of stem cells to generate a robust immune response against other types of cancer.
To investigate if stem cell-based vaccines are effective against hepatic tumors, we compared both HSC and ESC-derived whole-cell vaccines regards to the prevention of tumor establishment and shrinkage of existing tumors in a mouse model of implantable liver cancer. To minimize the impact of individual difference on the interpretation of results, we used the method of randomization and confirmed that there was no significant difference in either group before vaccination. We found that both HSC and ESC whole-cell vaccination was effective in preventing the outgrowth of implanted tumor cells in 100 and $90 \%$ of mice that were challenged one week after the last immunization. The benefits of prophylactic immunization appeared to wane over time, because the tumor burden in mice increased when the tumor challenge was administered four weeks after the last vaccination. It indicated that the immune memory was relatively weak in this study and thus the antitumor effect of prophylactic vaccination wanes overtime. Even so, both the rate of tumor formation and the average size of the subcutaneous tumors were still decreased significantly in the HSC vaccination group compared with the control group. In contrast, there were no statistically significant differences in the rate of tumor formation or in the average tumor size between the ESC vaccination group and the control group.

Although HSCs and ESCs share expression of several antigens, there was a question of whether HSCs would be better than ESCs at generating durable protection against established hepatoma. We discovered that immunization with an HSC-based vaccine in the absence of an adjuvant could result in the dynamic retardation or shrinkage of established liver cancer. In contrast, an ESC-based vaccine treatment led to a delayed progression of subcutaneous liver cancer but did not eliminate the tumor burden in this model. Thus we concluded that HSCs were the better option for a stem cell-based vaccine targeting liver cancer.

Although our results point to HSCs as being the better vaccine candidate, the precise mechanisms underlying the antitumor protection conferred by HSCs versus ESCs remain obscure. Recent evidence suggests that liver cancer cells express a subset of embryonic antigens that are downregulated during fetal development, and this downregulation occurs before the mammalian immune system determines 'self' versus 'non-self' (11). Embryonic antigens that are shared by both ESC and liver cancer cell might be included in the 'non-self' repertoire and remain immunogenic, leading to a robust antitumor immunity. ESC vaccination has been shown to increase the intratumor infiltration of $\mathrm{CD}^{+}$effector $\mathrm{T}$ cells, as well as decrease both $\mathrm{T}$ regulatory cells (Treg) and myeloid derived suppressor cells (MDSCs) within the tumor microenvironment (10-14). Further study indicated that the antitumor immunity conferred by ESC vaccination was primarily mediated by $\mathrm{CD} 8^{+} \mathrm{T}$ cells because depletion of this subset completely abrogated the protective effect in vivo. In addition, ESC vaccines can modulate the Th1/Th2 balance and tip the immune response towards Th1-driven antitumor immunity $(10-12)$.

Less is known regarding the effectiveness of HSCs as whole-cell vaccine candidates in the prophylaxis and treatment of liver cancer, or which antitumor mechanisms that HSCs induce are superior to ESCs. One possible explanation 
could be that hepatic tumor cells arise from the malignant transformation of normal HSCs and would thereby share the expression of stem cell-related pathways and antigens associated with immune escape mechanisms $(19,20)$. Vaccination with HSCs could elicit robust antitumor immunity against these malignantly transformed stem cells, since HSCs would present the identical immune targets that are expressed by the hepatic tumors (27). In this respect, antigens that were highly expressed in both HSC and liver cancer cells, such as $\alpha$-fetoprotein (AFP) and telomerase reverse transcriptase (TERT), have been shown to be frequently recognized by $\mathrm{T}$ cell and were capable of generating peptide-specific CTLs (6). However, HSC-derived whole-cell vaccines is not without potential risks and warrants a careful consideration for possible adverse side effects, although no mice showed signs of discomfort (such as the change of behavior, feeding, neuromuscular tone or appearance of fur) or died in this study. The most obvious risk would be cross-reactivity with normal HSCs in the liver, which could potentially lead to liver damage. However, previous studies have clearly demonstrated that the use of ESC and HSC did not result in significant autoimmune response in the context of immunization and transplantation (28), respectively. Since the potential side effect of autoimmunity of HSC vaccine has not been fully studied in the context of immunization, further studies are needed to investigate whether the antitumor immune responses elicited by HSC vaccination are vigorous enough to target the subset of HSCs present in the canals of Hering within the liver.

There were a few limitations in our study. First, we challenged immunized mice with a single hepatic cancer cell line (Hepa 1-6), so it is not known if HSC or ESC-based vaccines would produce the same antitumor protection against other liver cancer cell lines (especially cell lines derived from orthotopic liver cancer). Second, the size of the subcutaneous hepatic tumors was evaluated in our study, but less is known if reducing tumor size is associated with prolonged survival in vaccinated mice. Finally, the underlying antitumor mechanisms of HSC and ESC-derived whole-cell vaccines, as well as the potential toxicity of each type of vaccine are not fully illustrated in this study but it will be revealed in subsequent research.

In conclusion, recent developments in cancer vaccine design have explored the use of ESCs and HSCs as potential vaccine candidates, due to the expression of similar cell surface antigens by stem cells and cancer cells. Because effective treatments for patients with advanced-stage liver cancer are not currently available, a stem cell-based vaccine is one therapeutic strategy that may stimulate durable antitumor immunity and prolong patients' survival. Our study supports the hypothesis that HSCs are better than ESCs as a vaccine candidate for durable antitumor protection in a murine hepatocarcinoma model. We propose that HSCs are more effective than ESCs at generating long-lasting protection against hepatic tumors and could be used as both a prophylactic and therapeutic vaccine in the prevention and treatment of liver cancer.

\section{Acknowledgements}

The authors would like to thank the staff of the laboratory animal center in Fujian Medical University for providing excellent animal care. This study was supported by the Fujian Province Natural Science Fund (grant no. 2015J0105).

\section{References}

1. European Association for The Study of The Liver European Organisation for Research and Treatment of Cancer: EASL-EORTC clinical practice guidelines: Management of hepatocellular carcinoma. J Hepatol 56: 908-943, 2012.

2. International Agency for Research on Cancer (IARC): About CANCERMondial. http://www-dep.iarc.fr/. Accessed January 11, 2013.

3. Breous E and Thimme R: Potential of immunotherapy for hepatocellular carcinoma. J Hepatol 54: 830-834, 2011.

4. Greten TF, Manns MP and Korangy F: Immunotherapy of hepatocellular carcinoma. J Hepatol 45: 868-878, 2006.

5. Palmer DH, Midgley RS, Mirza N, Torr EE, Ahmed F, Steele JC, Steven NM, Kerr DJ, Young LS and Adams DH: A phase II study of adoptive immunotherapy using dendritic cells pulsed with tumor lysate in patients with hepatocellular carcinoma. Hepatology 49: 124-132, 2009.

6. Mizukoshi E, Nakamoto Y, Arai K, Yamashita T, Sakai A, Sakai Y, Kagaya T, Yamashita T, Honda M and Kaneko S: Comparative analysis of various tumor-associated antigen-specific T-cell responses in patients with hepatocellular carcinoma. Hepatology 53: 1206-1216, 2011

7. Mellman I, Coukos G and Dranoff G: Cancer immunotherapy comes of age. Nature 480: 480-489, 2011.

8. Butterfield LH, Ribas A, Dissette VB, Lee Y, Yang JQ, De la Rocha P, Duran SD, Hernandez J, Seja E, Potter DM, et al: A phase I/II trial testing immunization of hepatocellular carcinoma patients with dendritic cells pulsed with four alpha-fetoprotein peptides. Clin Cancer Res 12: 2817-2825, 2006.

9. Sawada Y, Yoshikawa T, Nobuoka D, Shirakawa H, Kuronuma T, Motomura Y, Mizuno S, Ishii H, Nakachi K, Konishi M, et al: Phase I trial of a glypican-3-derived peptide vaccine for advanced hepatocellular carcinoma: Immunologic evidence and potential for improving overall survival. Clin Cancer Res 18: 3686-3696, 2012.

10. Li Y, Zeng H, Xu RH, Liu B and Li Z: Vaccination with human pluripotent stem cells generates a broad spectrum of immunological and clinical responses against colon cancer. Stem Cells 27: 3103-3111, 2009.

11. Yaddanapudi K, Mitchell RA, Putty K, Willer S, Sharma RK, Yan J, Bodduluri H and Eaton JW: Vaccination with embryonic stem cells protects against lung cancer: Is a broad-spectrum prophylactic vaccine against cancer possible? PLoS One 7: e42289, 2012

12. Dong W, Du J, Shen H, Gao D, Li Z, Wang G, Mu X and Liu Q: Administration of embryonic stem cells generates effective antitumor immunity in mice with minor and heavy tumor load. Cancer Immunol Immunother 59: 1697-1705, 2010.

13. Mocan T and Iancu C: Effective colon cancer prophylaxis in mice using embryonic stem cells and carbon nanotubes. Int $\mathrm{J}$ Nanomed 6: 1945-1954, 2011.

14. Zhang ZJ, Chen XH, Chang XH, Ye X, Li Y and Cui H: Human embryonic stem cells - a potential vaccine for ovarian cancer. Asian Pac J Cancer Prev 13: 4295-4300, 2012.

15. Schoenhals M, Kassambara A, De Vos J, Hose D, Moreaux J and Klein B: Embryonic stem cell markers expression in cancers. Biochem Biophys Res Commun 383: 157-162, 2009.

16. Brewer BG, Mitchell RA, Harandi A and Eaton JW: Embryonic vaccines against cancer: An early history. Exp Mol Pathol 86: 192-197, 2009.

17. Ben-David U and Benvenisty N: The tumorigenicity of human embryonic and induced pluripotent stem cells. Nat Rev Cancer 11: 268-277, 2011.

18. Zhao W, Ji X, Zhang F, Li L and Ma L: Embryonic stem cell markers. Molecules 17: 6196-6236, 2012.

19. Mishra L, Banker T, Murray J, Byers S, Thenappan A, He AR, Shetty K, Johnson L and Reddy EP: Liver stem cells and hepatocellular carcinoma. Hepatology 49: 318-329, 2009.

20. Oishi N and Wang XW: Novel therapeutic strategies for targeting liver cancer stem cells. Int J Biol Sci 7: 517-535, 2011.

21. Akhurst B, Croager EJ, Farley-Roche CA, Ong JK, Dumble ML, Knight B and Yeoh GC: A modified choline-deficient, ethioninesupplemented diet protocol effectively induces oval cells in mouse liver. Hepatology 34: 519-522, 2001. 
22. Tirnitz-Parker JE, Tonkin JN, Knight B, Olynyk JK and Yeoh GC: Isolation, culture and immortalisation of hepatic oval cells from adult mice fed a choline-deficient, ethionine-supplemented diet. Int J Biochem Cell Biol 39: 2226-2239, 2007.

23. Dumble ML, Croager EJ, Yeoh GC and Quail EA: Generation and characterization of $\mathrm{p} 53$ null transformed hepatic progenitor cells: Oval cells give rise to hepatocellular carcinoma. Carcinogenesis 23: 435-445, 2002

24. Wang P, Liu T, Cong M, Wu X, Bai Y, Yin C, An W, Wang B, Jia J and You H: Expression of extracellular matrix genes in cultured hepatic oval cells: An origin of hepatic stellate cells through transforming growth factor beta? Liver Int 29: 575-584, 2009.
25. Conner DA: Mouse embryo fibroblast (MEF) feeder cell preparation. Curr Protoc Mol Biol. 2001; Chapter 23: Unit 23.2: May 1, 2001 (Epub ahead of primt). doi: 10.1002/0471142727. $\mathrm{mb} 2302 \mathrm{~s} 51$.

26. Xu C, Huang HR, Yu H, Zhao XL, Lu YL, Dai LC and Yao X: The study of separating murine embryonic fibroblast from embryonic stem cells by the differential adhesion method. Fen $\mathrm{Zi}$ Xi Bao Sheng Wu Xue Bao 39: 477-481, 2006 (In Chinese).

27. Dhodapkar MV and Dhodapkar KM: Vaccines targeting cancer stem cells: Are they within reach? Cancer J 17: 397-402, 2011.

28. Oertel M: Fetal liver cell transplantation as a potential alternative to whole liver transplantation? J Gastroenterol 46: 953-965, 2011. 